\title{
BIODIESEL REACTION SCREENING USING OSCILLATORY FLOW MESO REACTORS
}

\author{
M. Zheng, R. L. Skelton and M. R. Mackley* \\ Department of Chemical Engineering, University of Cambridge, Cambridge, UK.
}

\begin{abstract}
This paper is concerned with performing laboratory scale alkali-catalyst transesterification for biodiesel reaction and ranking conversion and performance between a stirred tank reactor, and oscillatory flow meso reactors in both batch and continuous mode. The results show that a comparative conversion can be achieved for the same reaction conditions, thereby demonstrating that batch and continuous meso reactor configurations can be used for a high throughput screening biodiesel reactions.
\end{abstract}

Keywords: biodiesel; oscillatory flow; meso reactor; screening.

\section{INTRODUCTION}

Biodiesel is promoted as a sustainable alternative to petroleum diesel and is characterized as the methyl or ethyl esters of long chain of fatty acids derived from vegetable oils or animal fats. Transesterification is currently the predominant chemical process to produce biodiesel, often referred to as fatty acid methyl ester (FAME), by reacting vegetable oil with methanol in presence of an alkaline catalyst. Advantages of using biodiesel or blended biodiesel with petroleum diesel as a fuel include lower overall life cycle $\mathrm{CO}_{2}$ emissions, and reduced air pollution (Choi and Oh, 2006; Fernando et al., 2006; Peng et al., 2006; Tan et al., 2004).

\section{Biodiesel Production}

The transesterification reaction in which the triglyceride molecule reacts with alcohol, e.g., methanol to form three FAME molecules and one glycerol molecule includes three consecutive steps of reversible reactions (Freedman et al., 1986; Schwab et al., 1987).

$$
\begin{aligned}
\text { Triglyceride }+\mathrm{MeOH} \leftrightarrow & \text { Diglyceride } \\
& + \text { FAME }
\end{aligned}
$$

Diglyceride $+\mathrm{MeOH} \leftrightarrow$ Monoglyceride

$$
+ \text { FAME }
$$

Monoglyceride $+\mathrm{MeOH} \longleftrightarrow$ Glycerol + FAME (3)
The overall reaction can be written as

$$
\begin{aligned}
& \mathrm{CH}_{2}-\mathrm{OOC}-\mathrm{R}_{1} \\
& \mathrm{CH}-\mathrm{OOC}-\mathrm{R}_{2}+3 \mathrm{CH}_{3} \mathrm{OH} \\
& \begin{array}{l}
\mathrm{CH} \\
\text { Triglyceride }
\end{array} \\
& \mathrm{R}_{1}-\mathrm{COO}-\mathrm{CH}_{3} \quad \mathrm{CH}_{2}-\mathrm{OH} \\
& \mathrm{R}_{2}-\mathrm{COO}-\mathrm{CH}_{3}+\mathrm{CH}-\mathrm{OH} \\
& \mathrm{R}_{3}-\mathrm{COO}-\mathrm{CH}_{3} \quad \mathrm{CH}_{2}-\mathrm{OH} \\
& \text { Methyl easters } \quad \mathrm{Glycerol}
\end{aligned}
$$

In practice, since all three reactions are reversible, a significant excess of methanol with catalyst is necessary to obtain a high conversion. A molar ratio of $6: 1$ of methanol to vegetable oil gives maximum conversion using $1 \%$ sodium hydroxide (Freedman et al., 1984, 1986; Ma and Hanna, 1999). Either acid or alkali, such as sodium hydroxide, sodium methoxide, and potassium hydroxide can be used as catalysts. Sodium methoxide has an advantage that it does not produce water and thus prevents saponification side reactions, although it is more expensive and requires greater care in handling (Freedman et al., 1984; Vicente et al., 2004). A catalyst concentration of $0.5 \mathrm{~mol} \%$ sodium methoxide and up to $2 \mathrm{~mol} \%$ sodium hydroxide was suggested by. Schuchardt et al. (1998) Studies have also been carried out to investigate alternative heterogeneous catalysts (Kim et al., 2004) and enzymes (Jackson and King, 1996) for biodiesel production. 
The production of biodiesel can either be conducted in a batch process or continuous operation (Ma and Hanna, 1999). Continuous processes can be more efficient, in terms of overall reaction cycle times, cost and product quality control.

Biodiesel is produced at various scales from what amounts to back yard operations using agitated vessels or pump round mixing followed by settling and purification in the same vessel to large scale continuous plant. Some medium scale operations also use batch processing usually with a separate purification train. Most continuous plants use a combination of static in line mixers followed by long tubular reactors and complex purification trains, often involving distillation. The Cambridge OFM reactor is designed for medium scale continuous operations and can be skid mounted for ease of transport. It combines many of the advantages of the batch and tubular reactor and is very compact.

With few exceptions the process takes place at about $60^{\circ} \mathrm{C}$ and just above ambient pressure to ensure that the methanol stays in the liquid phase. In the continuous plants the reactants are normally pre-heated but in the batch process heating is usually carried out in the reaction vessel. The most important factor in biodiesel production tends to be mass transfer rather than heat transfer as it is vital to get the reactants, which have widely differing viscosities mixed as rapidly as possible. Once the first step in the reaction is initiated the methanol will dissolve in the products hence facilitating the subsequent steps.

The usual way of trialling biodiesel reactions and reactors is currently to conduct small scale batch reactions in stirred tank followed by larger scale pilot and continuous production reactors. In this paper a 'meso scale' $5 \mathrm{ml}$ batch and a continuous reactor were used with an initial object to show performance comparison with stirred laboratory scale batch reactor performance and also demonstrate proof of concept for the continuous meso reactor. The use of the batch meso reactor will allow screening of potential feedstocks and catalysts for a small scale than even stirred batch laboratory reactor. The continuous meso reactor has the potential to screen many continuous processing reaction conditions; establish optimal conditions and in principle the reactor configuration could be scaled up without further trials for a large scale biodiesel production.

\section{The Oscillatory Flow Reactor}

The oscillatory flow mixing (OFM) is a process intensification technology to improve mixing as well as other process properties, such as heat transfer (Mackley and Stonestreet, 1995; Stephens and Mackley, 2002), mass transfer (Mackley et al., 1998; Ni et al., 1995), plug flow residence time distribution (RTD) (Howes et al., 1991; Mackley and Ni, 1993; Mackley et al., 1996), and multiphase suspensions (Mackley et al., 1993; Ni et al., 2003). The oscillatory flow reactor (OFR) is a tubular reactor containing periodically spaced low orifice constrictions where the fluid oscillations are applied in an axial direction within the tube as shown in Figure 1. A macro-scale OFR containing sharp periodic baffles (SPB-OFR, or baffled OFR) was developed for a wide variety of reactions (Chew et al., 2004; Fabiyi and Skelton, 2000; Harvey et al., 2001; Lee et al., 2002; Ni et al., 2001) including the biodiesel production reaction (Harvey et al., 2003).

In the OFR with sharp periodic baffles, the interaction of the orifice baffles with the oscillating fluid causes periodic toroidal vortices between each baffle (Howes et al., 1991; Ni et al., 2002). Applying net flow in this OFR, the residence time distribution can be close to that of plug flow (Stonestreet and Van der Veeken, 1999). Unlike conventional plug flow reactors in which the plug flow is caused by turbulent net flow, the plug flow achieved in an SPB-OFR is obtained by the oscillation conditions. This offers the SPB-OFR an important process intensification feature that it allows 'long' (e.g., greater than $15 \mathrm{~min}$ ) reactions to be performed in a plug flow reactor of relatively small length-to-diameter ratio (Harvey et al., 2001).

Recently, a meso-scale OFR containing smooth periodic constrictions (SPC-OFR, or meso reactor) as shown in Figure 1(b) was developed at Cambridge for the scaledown pharmaceutical and biological screenings and production. Similar periodic vortex shedding flow patterns (Reis et al., 2005) and a near plug flow RTD (Reis et al., 2004) were observed within the meso reactor as in SPBOFR. A continuous meso reactor was developed to carry out multiphase reactions (Reis et al., 2006) such as biodiesel reaction in lab scale.

The oscillatory Reynolds number, $R e_{\mathrm{o}}$, and Strouhal number, $S t$, characterize the hydrodynamics within an OFR to describe the oscillatory flow as well as the geometrical configuration of the baffles. The $R e_{o}$ and the $S t$, are defined
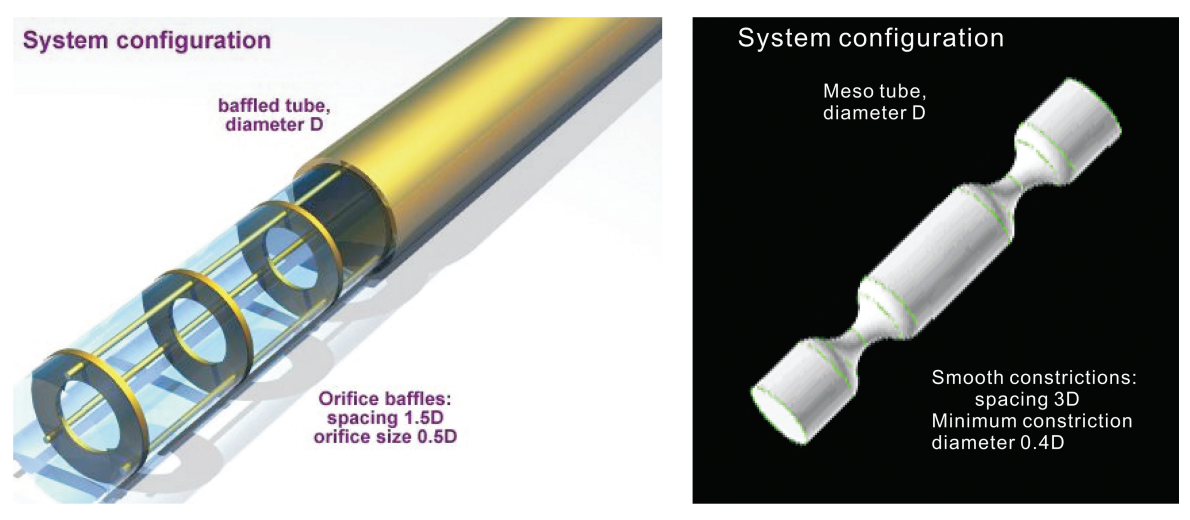

Figure 1. The configuration of oscillatory flow reactors (OFR). 
as follows:

$$
\begin{aligned}
& R e_{\circ}=\frac{2 \pi f x_{\circ} \rho D}{\mu} \\
& S t=\frac{D}{4 \pi x_{0}}
\end{aligned}
$$

where $x_{\mathrm{o}}$ is the centre to peak amplitude of oscillation; $f$ is oscillatory frequency $(\mathrm{Hz}) ; D$ is the tube diameter $(\mathrm{m}) ; \rho$ is fluid density $\left(\mathrm{kg} \mathrm{m}^{-3}\right)$ and $\mu$ is fluid viscosity (Pa s). The oscillatory Reynolds number describes the intensity of mixing applied to the tube, where $2 \pi f x_{\mathrm{o}}$ represents the maximum oscillatory velocity $\left(\mathrm{m} \mathrm{s}^{-1}\right)$; the Strouhal number is the ratio of the tube diameter to the oscillatory amplitude. A net flow Reynolds number, $R e_{\mathrm{n}}$, becomes relevant for the case where there is an additional net flow along the tube, described as follows:

$$
R e_{\mathrm{n}}=\frac{\rho \mu D}{\mu}
$$

where $u$ is the superficial net flow velocity through the tube $\left(\mathrm{m} \mathrm{s}^{-1}\right)$.

In this paper, a biodiesel reaction was carried out in a batch and continuous meso reactor, and the reaction kinetics was compared with a standard laboratory stirred vessel reactor. Effective oscillation conditions were established in the batch meso reactor and reaction conversions were studied in all three types of reactors.

\section{EXPERIMENTAL}

\section{Experimental Materials}

Refined vegetable oil (Holland UK Ltd) with a density of $0.894 \mathrm{~g} \mathrm{ml}^{-1}$ was used as the source of triglyceride. The viscosity was $0.06 \mathrm{~Pa}$ s at $20^{\circ} \mathrm{C}$ and $0.02 \mathrm{~Pa}$ s at $60^{\circ} \mathrm{C}$ measured by a controlled strain rheometer (Rheometric Scientific, Ares). The methanol/catalyst phase was $4.2 \%$ (wt/wt) sodium methoxide (Sigma-Aldrich) in methanol (Fluka, Germany) solution.
The volume ratio of oil and alcohol solution was $4: 1$, which corresponded to $1: 6$ in molar ratio. The reaction was carried out at $60^{\circ} \mathrm{C}$ for all three types of reactors.

\section{Experimental Methods and Reactor Configurations}

Batch stirred reactor

A $42 \mathrm{~mm}$ internal diameter and $57 \mathrm{~mm}$ depth glass vessel with a circulation outer jacket was used as the batch stirred reactor. The solution held inside the reactor was stirred using a $25 \mathrm{~mm} \times 7 \mathrm{~mm}$ magnetic stirrer at the bottom of the vessel. The rotation speed of the stirrer was maintained at $200 \mathrm{rpm}$, Reynolds number $R e_{\mathrm{i}}=444$, using a hot plate with a digital rotation control. The impeller Reynolds number was estimated by (Oldshue, 1983)

$$
R e_{\mathrm{i}}=\frac{\rho N D_{\mathrm{i}}^{2}}{\mu}
$$

where $N$ is the stirring speed (rps), $D_{i}$ is impeller diameter $(\mathrm{m})$. A schematic diagram of the batch stirred reactor is shown in Figure 1 below.

Eight millilitres of methanol/catalyst solution was added into $32 \mathrm{ml}$ preheated vegetable oil in a glass stirred reactor as shown in Figure 2. The temperature was controlled at $60^{\circ} \mathrm{C}$ by circulating hot water via a stirred thermostatic pump (Grant, Cambridge, UK) fitted in a water bath.

Reactions were carried out for $40 \mathrm{~min}$. Samples of $0.25 \mathrm{ml}$ were obtained at 5 min intervals from the beginning of reaction and transferred into a $2 \mathrm{ml}$ centrifuge tube. The samples were quenched by adding $0.25 \mathrm{ml}$ of $0.1 \mathrm{~N}$ hydrochloric acid to stop reaction and then centrifuged (Beckman, USA, TJ-6). About $0.15 \mathrm{ml}$ clear top phase were removed into a clean centrifuge tube for analysis in GC. The experiments were carried out three times to obtain repeat samples for each time point.

\section{Batch oscillatory flow meso reactor}

The batch oscillatory meso reactor consists of a Smooth Periodic Constriction (SPC) tube, an oscillator and a metal

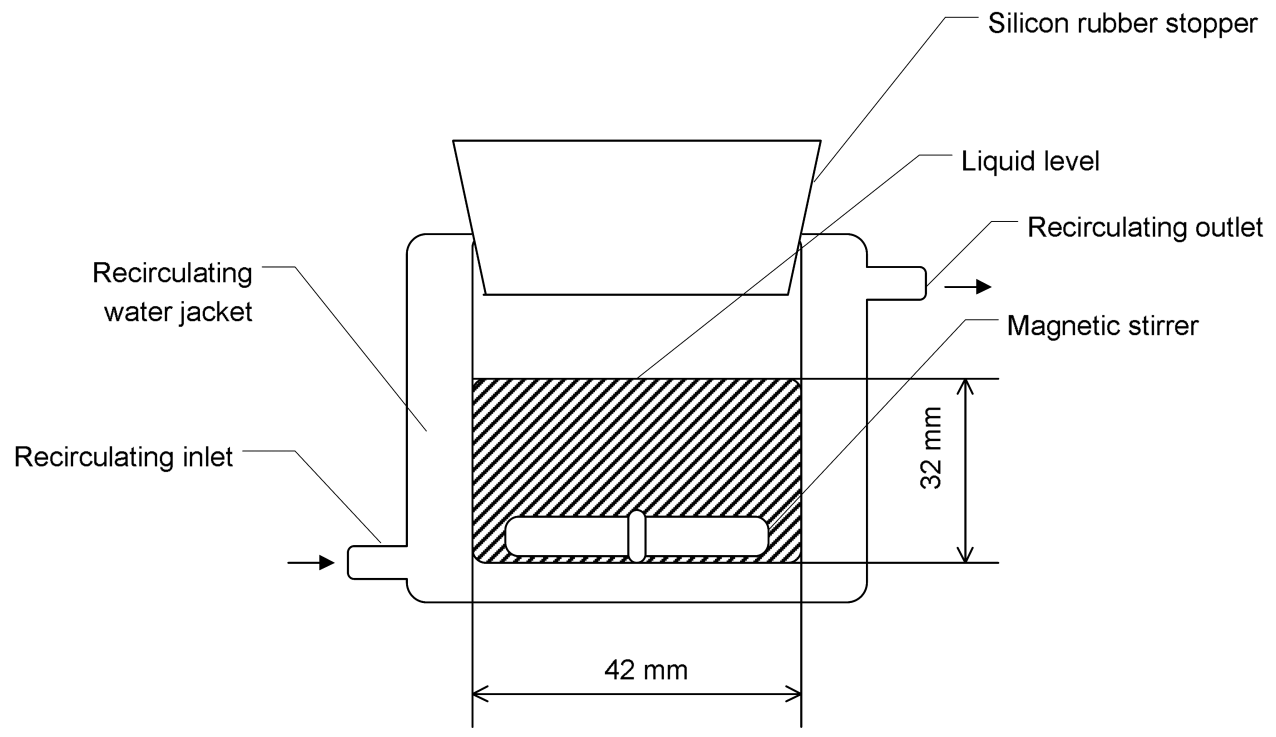

Figure 2. The schematic diagram of batch stirred reactor.

Trans IChemE, Part B, Process Safety and Environmental Protection, 2007, 85(B5): 1-8 


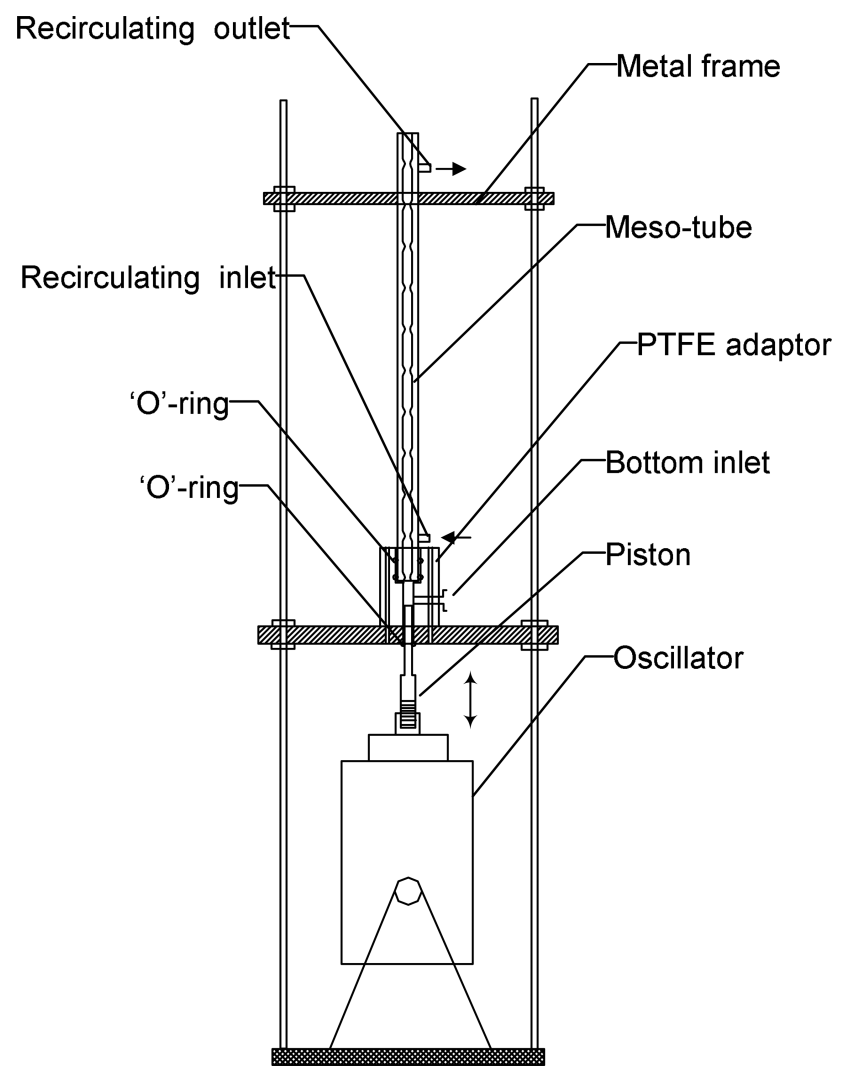

Figure 3. A schematic diagram of batch meso reactor.

fitting frame (as shown in Figure 3). A vertically mounted SPC (meso-) tube $(20 \mathrm{~cm}$ long, $5 \mathrm{~mm}$ maximum inner diameter glass tube with a heating/cooling jacket) was shown in Figure 4.

The inner glass wall was periodically 'pinched' to produce smooth periodic constrictions. The spacing, $\lambda$, between any two constrictions was $15.2 \mathrm{~mm}$ (approximately three times the tube diameter) and the length, $\delta$, of each constriction was $6.4 \mathrm{~mm}$. The minimum diameter, $h$, of the constricted zone was $1.6 \mathrm{~mm}$, representing $10 \%$ of the maximum cross-sectional area. The total volume of the meso-tube is $4.8 \mathrm{ml}$.

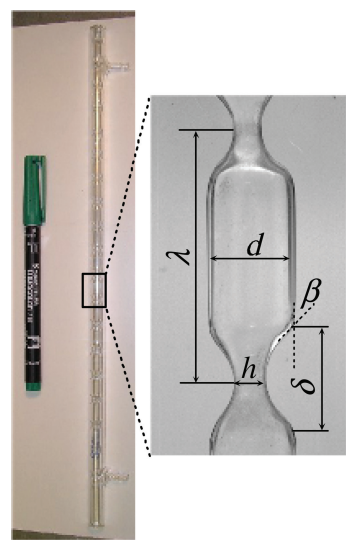

Figure 4. The geometry of a meso tube $(d=5.0 \mathrm{~mm}, \lambda=15.2 \mathrm{~mm}$, $h=1.6 \mathrm{~mm}, \delta=6.4 \mathrm{~mm}, \beta \approx 45^{\circ}$ ).
A vertical jacketed meso-tube and an electromagnetic oscillator (LDS, UK, V406) were connected using a PTFE fitting fixed to a metal frame, as shown in Figure 4. A signal generator (LDS, UK, PO100) and amplifier (LDS, UK, PA25E) controlled the amplitude and frequency of oscillation. Typical oscillation frequencies and amplitudes used were between 0 and $20 \mathrm{~Hz}$ and 0 and $3 \mathrm{~mm}$ (centre-to-peak) respectively.

The reaction was carried out at $60^{\circ} \mathrm{C}$ by circulating hot water via a stirred thermostatic pump (Grant, Cambridge, UK) fitted in a water bath. $3.8 \mathrm{ml}$ vegetable oil was injected into the pre-heated meso tube from the bottom inlet, and started oscillation for 2 min to obtain a uniform temperature. $0.95 \mathrm{ml} \mathrm{methanol} /$ catalyst solution was added into the meso tube via a $1 \mathrm{ml}$ syringe with a $10 \mathrm{~cm}$ needle when the oil was oscillating.

Different oscillation conditions and reaction time were carried out to screen for optimal oscillation conditions in the batch meso reactor. The detail of experimental conditions was listed in Table 1.

Samples were collected at the end of the reaction after 30 or $40 \mathrm{~min}$ by a $10 \mathrm{ml}$ syringe via a $20 \mathrm{~cm}$ needle, and transferred into a $50 \mathrm{~mm} \times 19 \mathrm{~mm}$ glass vial. The sample was quenched immediately and centrifuged. One millilitre of top clear phase was removed into a $3 \mathrm{ml}$ vial for $\mathrm{GC}$ analysis.

The optimal oscillation condition was investigated by comparing the final Triglyceride conversions at different oscillation frequency and amplitude. The reaction was then carried out under this condition to study the concentration change with time. Samples were collected from each batch with the reaction time of 5, 10, 20, 30 and $40 \mathrm{~min}$. Three experiments were carried out for each time interval. The samples were centrifuged and analysed by GC (see later for details).

\section{Continuous oscillatory flow meso reactor}

Continuous flow experiments were carried out using an apparatus shown in Figure 5. The apparatus consisted of a product vessel, a mixing unit, syringe pumps, feed tanks, and 8 meso tubes (with a tube length of $730 \mathrm{~cm}$ ) with U-bends, providing a total volume of $103 \mathrm{ml}$. The experimental oscillation conditions were selected as oscillation frequency $10 \mathrm{~Hz}$ and amplitude $2 \mathrm{~mm}$ (centre to peak).

Vegetable oil was filled into a $1 \mathrm{I}$ glass feed tank and the methanol/catalyst solution was added from a $500 \mathrm{ml}$ glass feed tank, which were both equipped with a magnetic stirrer and a feedback temperature controlled hot plate. A controllable feed from the tanks was produced using two double acting syringe pumps (Pharmacia, P500) which feed both components to the reactor mixing head that was immediately above the oscillator. The flowrate of vegetable oil was $101 \mathrm{ml} \mathrm{h}^{-1}$, and that of methanol solution was $25 \mathrm{ml} \mathrm{h}^{-1}$,

Table 1. Oscillation conditions for maximum conversion screening.

\begin{tabular}{lcrrr}
\hline $\begin{array}{l}\text { Oscillation } \\
\text { frequency }(\mathrm{Hz})\end{array}$ & $\begin{array}{c}\text { Oscillation } \\
\text { amplitude }(\mathrm{mm})\end{array}$ & $R e_{\mathrm{o}}$ & $\mathrm{St}$ & $\begin{array}{c}\text { Reaction time } \\
(\mathrm{min})\end{array}$ \\
\hline 10 & 1 & 67 & 0.4 & $30 / 40$ \\
8 & 2 & 107 & 0.2 & $30 / 40$ \\
10 & 2 & 134 & 0.2 & $30 / 40$ \\
12 & 2 & 161 & 0.2 & 40 \\
6 & 3 & 120 & 0.13 & $30 / 40$ \\
15 & 3 & 301 & 0.13 & 40 \\
\hline
\end{tabular}




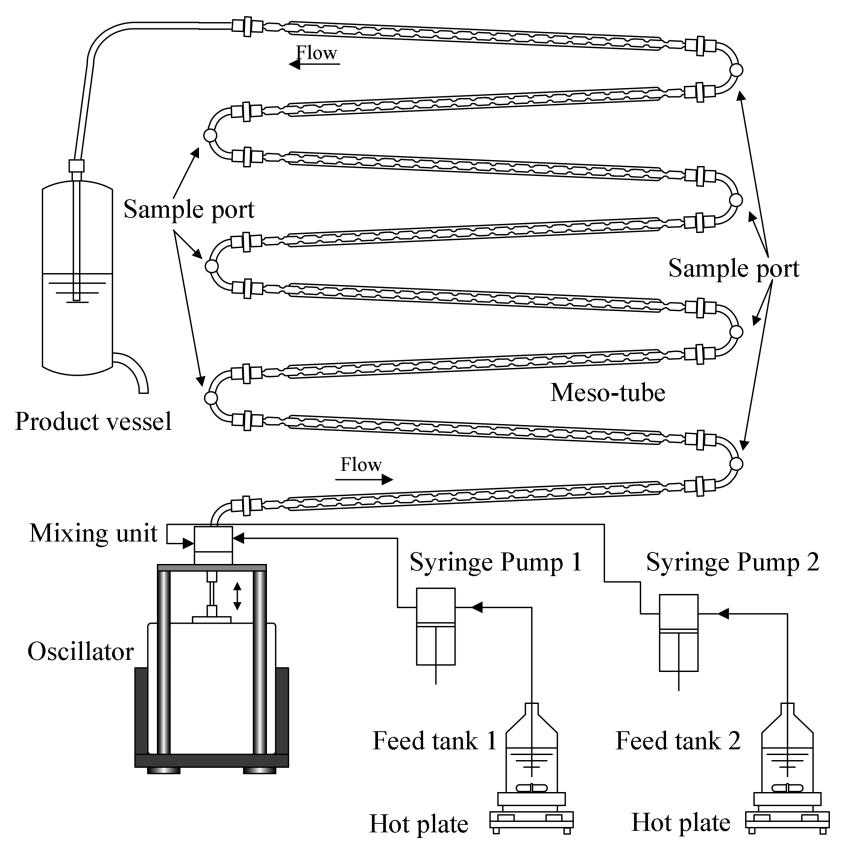

Figure 5. A schematic diagram of continuous meso reactor.

which provided a mean residence time of $49 \mathrm{~min}$. The temperature of meso tubes and product vessel was controlled at $60^{\circ} \mathrm{C}$. After $100 \mathrm{~min}$ of operation, about $0.25 \mathrm{ml}$ samples were taken from each of the seven sample ports (as shown in Figure 5) and the final sample was collected from the product vessel. Three sets of samples were taken after further 30 min intervals for each sample position. Samples were quenched with $0.1 \mathrm{~N} \mathrm{HCl}$ and centrifuged immediately and the top layer was separated for GC analysis.

\section{Analytical Methods}

An automatic 'cool on column' injection Gas Chromatograph (GC, Angilent Technologies, USA, 6890) was used for analysis of concentration of product samples from the reaction in accordance with British Standard EN14205. A capillary GC column (Angilent Technologies, USA, DB-1HT) with $0.32 \mathrm{~mm}$ i.d. was used in the apparatus. The temperature of flame ionization detector was $380^{\circ} \mathrm{C}$, and carrier gas pressure was $80 \mathrm{kPa}$. The analysis of biodiesel for each sample was carried out by sylating then dissolving $100 \mathrm{mg}$ of biodiesel sample in $8 \mathrm{ml}$ of heptane and injecting $1 \mu \mathrm{L}$ of this solution in GC. Two internal standards are used, one for glyverol and one for the glycerides. The monoglyceride (MG), diglyceride (DG), triglyceride (TG) contents were expressed as weight percent $(\mathrm{w} / \mathrm{w} \%)$. The instrument was calibrated using glycerol, mooolein, diolein and triolein in n-heptane solutions in accordance with BS EN 14105. The viscosity of FAME was measured by the Ares rheometer (Rheometric Scientific) at $20^{\circ} \mathrm{C}$ and $60^{\circ} \mathrm{C}$.

\section{RESULTS AND DISCUSSION \\ Batch Stirred Reactor}

The concentration change of monoglyceride (MG), diglyceride (DG), triglyceride (TG) contents with the reaction time is shown in Figure 6. The data shows that $98 \%$ of the

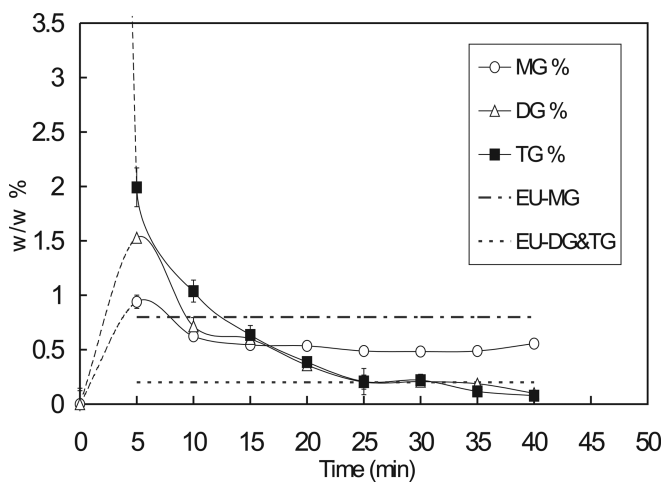

Figure 6. Concentration of Mono-, dl-, triglyceride contents (wt/wt\%) in batch stirred reactor at impeller speed $=200 \mathrm{rpm}$ and temperature $=60^{\circ} \mathrm{C}$.

reaction occurs within $10 \mathrm{~min}$, however, in order to reach adequately low DG and TG concentration (allowed by the EU standard EN14214), a total reaction time of order 35 minutes is required. Figure 6 shows that after $15 \mathrm{~min}$ reaction, the concentration of MG remains at $0.5 \%$ until the end of reaction. The concentrations of DG and TG continue to decrease as the reaction proceeds. This results show that each of the three step reaction [equations (1)-(3)] moves forward; after about $10 \mathrm{~min}$ the overall reaction rate of reaction (2) is faster than that of reaction (1), and almost equivalent to that of reaction (3). Figure 6 also shows after $40 \mathrm{~min}$ the amount of MG, DG and TG are all acceptable by the EU standard EN14214.

\section{Oscillatory Flow Meso Reactor}

\section{Mixing conditions}

In order to establish satisfactory mixing conditions within an oscillatory flow meso reactor, a series of experiments were carried out (see Table 1) and it was found that a minimum of 40 min reaction time and an effective mixing condition were necessary to achieve a high conversion of triglyceride allowed by EU Standard EN14214. The final concentrations of TG after 30/40 min reaction time are shown in Figure 7.

The data shows that in order to achieve the conversion allowed by the EU standard, a combination of oscillation

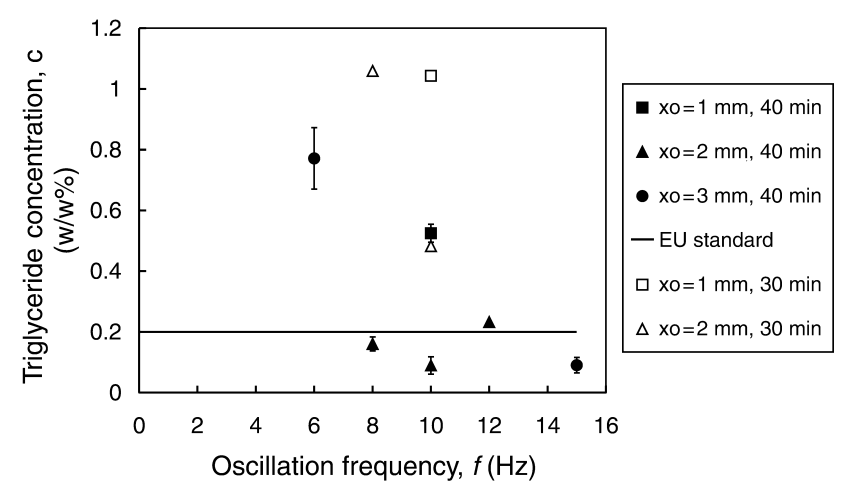

Figure 7. Effect of oscillation conditions on triglyceride conversion after 30 and $40 \mathrm{~min}$. 
frequency $8 \mathrm{~Hz}$ and amplitude $2 \mathrm{~mm}$ was required, which corresponds to $R e_{o}>107$, and $S t<0.2$. At this oscillatory Reynolds number, the flow separation occurs after each constriction within the meso tube (Reis et al., 2005). It shows that the fluid oscillation conditions control the vortex mixing intensity, and therefore influence the droplet breakup and emulsion formation in the initial two phase system. The results show that the mass transfer between the oil phase and methanol phase was enhanced at higher oscillation frequency and amplitude during the reaction. In order to achieve the required conversion with minimum energy input, $10 \mathrm{~Hz}$ and $2 \mathrm{~mm}$ was selected as the oscillation condition for the biodiesel reaction in both batch and continuous meso reactor.

\section{Batch meso reactor}

The concentration changes of monoglyceride (MG), diglyceride (DG), triglyceride (TG) contents with the reaction time within the batch oscillatory flow meso reactor are shown in Figure 8. The concentration responses and the overall conversion of TG obtained in batch meso reactor were similar to those in the batch stirred reactor. However the consumption of $T G$, and generation of $D G$ and $M G$ were slower in the batch meso reactor than batch stirred reactor in the first $5 \mathrm{~min}$, and there was a stepper change of TG concentration curve between 10-30 min while it was linear in batch stirred reactor. In general, there is little difference between the conversion kinetics in the batch stirred reactor and batch meso reactor.

\section{Continuous meso reactor}

In the experiments carried out in the continuous meso reactor, the oscillatory frequency was $10 \mathrm{~Hz}$, and amplitude was $2 \mathrm{~mm}$, corresponding to the oscillatory Reynolds number $R e_{\mathrm{o}}=134$ and Strouhal number $S t=0.2$. At the experimental condition, a net flow Reynolds number $R e_{n}=1.9$ was achieved, giving a ratio of $\psi=R e_{\mathrm{o}} / R e_{\mathrm{n}}=70$. Previous studies showed that the oscillatory flow dominated the system at this operating condition, so that the net flow did not affect the fluid oscillations and mixing (Harvey et al., 2001; Stonestreet and Van der Veeken, 1999). The results showed that the net flow was decoupled with mixing, and the reaction could be carried out within the reactor using the

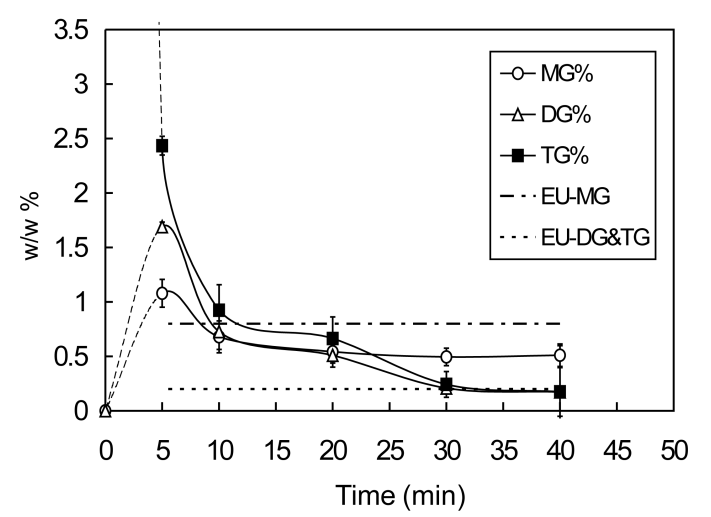

Figure 8. Concentration of Mono-, di-, triglyceride contents (wt/wt\%) in batch meso reactor at oscillation frequency $=10 \mathrm{~Hz}$, amplitude = $2 \mathrm{~mm}$ and temperature $=60^{\circ} \mathrm{C}$.

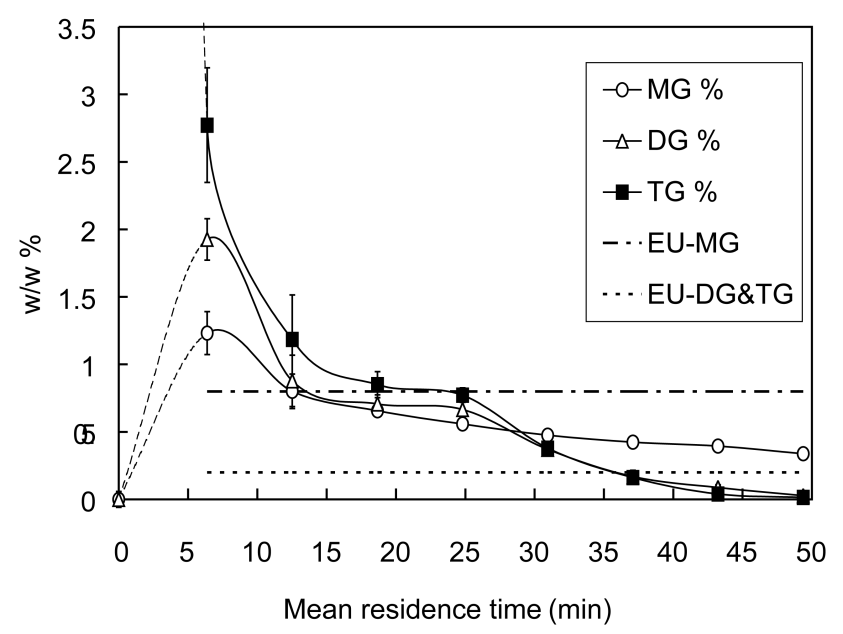

Figure 9. Concentration of Mono-, di-, triglyceride contents (wt/ $w \mathrm{w}$ ) in continuous meso reactor at oscillation frequency $=10 \mathrm{~Hz}$, amplitude $=2 \mathrm{~mm}$, total net flowrate $=126 \mathrm{ml} \mathrm{h}^{-1}$ and temperature $=60^{\circ} \mathrm{C}$.

oscillation for mixing and thereby enabling the net flow to provide an independent control of residence time.

For the continuous biodiesel reaction in the meso reactor, samples were taken at different positions along the tube, thereby obtaining the conversions as a function of different residence times. The concentration changes of each component with mean residence time are shown in Figure 9. The trend of conversion versus time in the continuous flow meso reactor was found to be similar to the conversion kinetics observed in the batch meso reactor (Figure 8 ) and stirred reactor (Figure 6). This demonstrated that the continuous meso reactor conversion kinetics matched that of batch reactor and that axial dispersion in the flow reactor did not appear to have a significant effect on conversion. For the continuous meso reaction, after $40 \mathrm{~min}$ reaction time, both TG and DG concentration were below EU standard upper limits, and the DG and TG concentration was lower than the concentration at equivalent reaction time in the batch meso reactor.

It would have been useful to evaluate power densities for both the stirred vessel and Smooth Periodic Constriction meso tube reactor. Correlations exit for both impeller stirring reactors (den Hollander et al., 2002; Oldshue, 1983) and also Sharp Edge Baffles OFM units (Ni et al., 1995, 2003); however in one case a magnetic bar stirrer was used for the batch reactor and in another case the meso tube contains smooth periodic constrictions, at present power density correlations do not exist for both cases.

\section{CONCLUSIONS}

This paper has shown that at a laboratory scale a transition from (1) batch stirred to (2) batch meso to (3) continuous meso reactor can be made where for the same reactants, reaction time and temperature the results show an equivalent conversion.

The conversion kinetics of the reaction described in Figures 6,8 and 9 all show that the bulk of the reaction occurs within $10 \mathrm{~min}$, however in order to obtain an EU standard of DG and TG concentration, it is necessary to continue the reaction for a further $30 \mathrm{~min}$. From optical observation of 
the reactor showed that the initial early stages of conversion appeared to be two phases, whereas at the later stages, e.g., mean residence time $t>2.5 \mathrm{~min}$, the reaction fluid was optically homogeneous. This indicates that the reactor design could be divided into two stages. Initial intense mixing is required for the first stage of reaction where the fluid is a two phase system, however as the reaction continues less intense mixing is necessary in order to obtain a homogeneous reactant.

The fact that a similar conversion was achieved between the batch meso reactor and continuous meso reactor suggests that adverse dispersion along the reactor tube does not occur, because if significant dispersion was present the conversion kinetics would be different (Busto et al., 2006). The results demonstrate that the meso reactor can be used for continuous reaction on a laboratory scale. Previous studies (Smith and Mackley, 2006) have demonstrated scaling in an oscillatory flow sharp edge baffled tube can be easily scaled up. The fluid mechanics within a meso reactor is similar to that within a baffled tube, therefore successful reaction conditions established in a meso reactor should be able to be translated to larger scale oscillatory flow baffled reactor.

\section{REFERENCES}

Busto, M., D'lppolito, S.A. et al., 2006, Influence of the axial dispersion on the performance of tubular reactors during the noncatalytic supercritical transesterification of triglycerides, Energy \& Fuels, 20(6): 2642-2647.

Chew, C.M., Ristic, R.I. et al., 2004, Crystallization of paracetamol under oscillatory flow mixing conditions, Crystal Growth \& Design, 4(5): 1045-1052.

Choi, S.H. and Oh, Y, 2006, The emission effects by the use of biodiesel fuel, International Journal of Modern Physics B, 20(25-27): 4481-4486.

den Hollander, J.L., Zomerdijk, M. et al., 2002, Continuous enzymatic penicillin $\mathrm{G}$ hydrolysis in countercurrent water-butyl acetate biphasic systems, Chem Eng Sci, 57(9): 1591-1598.

Fabiyi, M.E. and Skelton, R.L., 2000, On the effect of pulsing conditions on reaction rates in a pulsed baffled tube photoreactor (PBTPR), Process Safety and Environmental Protection, 78(B5): 399-404.

Fernando, S., Hall, C. et al., 2006, NOx reduction from biodiesel fuels, Energy \& Fuels, 20(1): 376-382.

Freedman, B., Butterfield, R.O. et al., 1986, Transesterification kinetics of soybean oil, Journal of the American Oil Chemists Society, 63(10): 1375-1380.

Freedman, B., Pryde, E.H. et al., 1984, Variables affecting the yields of fatty esters from transesterified vegetable-oils, Journal of the American Oil Chemists Society, 61(10): 1638-1643.

Harvey, A.P., Mackley, M.R. et al., 2003, Process intensification of biodiesel production using a continuous oscillatory flow reactor, Journal of Chemical Technology and Biotechnology, 78(2-3): 338-341.

Harvey, A.P., Mackley, M.R. et al., 2001, Operation and optimization of an oscillatory flow continuous reactor, Ind Eng Chem Res, 40(23): 5371-5377

Howes, T., Mackley, M.R. et al., 1991, The simulation of chaotic mixing and dispersion for periodic flows in baffled channels, Chem Eng Sci, 46(7): 1669-1677.

Jackson, M.A. and King, J.W., 1996, Methanolysis of seed oils in flowing supercritical carbon dioxide, Journal of the American Oil Chemists Society, 73(3): 353-356.

Kim, H.-J., Kang, B.-S. et al., 2004, Transesterification of vegetable oil to biodiesel using heterogeneous base catalyst, Catal Today, 93-95: 315-320.

Lee, C.T., Buswell, A.M. et al., 2002, The influence of mixing on lysozyme renaturation during refolding in an oscillatory flow and a

Q1 stirred-tank reactor, Chem Eng Sci, 57(10): 1679-1684.
Ma, F.R. and Hanna, M.A., 1999, Biodiesel production: a review, Bioresource Technology, 70(1): 1-15.

Mackley, M.R. and Ni, X., 1993, Experimental fluid dispersion measurements in periodic baffled tube arrays, Chem Eng Sci, 48(18): 3293-3305

Mackley, M.R., Smith, K.B. et al., 1993, The mixing and separation of particle suspensions using oscillatory flow in baffled tubes, Chem Eng Res Des, 71(A6): 649-656.

Mackley, M.R. and Stonestreet, P., 1995, Heat transfer and associated energy-dissipation for oscillatory flow in baffled tubes, Chem Eng Sci, 50(14): 2211-2224.

Mackley, M.R., Stonestreet, P. et al., 1996, Residence time distribution enhancement in reactors using oscillatory flow, Chem Eng Res Des, 74(A5): 541-545.

Mackley, M.R., Stonestreet, P. et al., 1998, Evaluation of a novel selfaerating, oscillating baffle column, Canadian Journal of Chemical Engineering, 76(1): 5-10.

$\mathrm{Ni}, \mathrm{X}$., Cosgrove, J.A. et al., 2001, Experimental study of flocculation of bentonite and Alcaligenes eutrophus in a batch oscillatory baffled flocculator, Chem Eng Res Des, 79(A1): 33-40.

$\mathrm{Ni}, \mathrm{X} ., \mathrm{Gao}, \mathrm{S}$. et al., 1995, A comparative-study of mass-transfer in yeast for a batch pulsed baffled bioreactor and a stirred-tank fermenter, Chem Eng Sci, 50(13): 2127-2136.

$\mathrm{Ni}$, X., Jian, H. et al., 2002, Computational fluid dynamic modelling of flow patterns in an oscillatory baffled column, Chem Eng Sci, 57(14): 2849-2862.

Ni, X., Mackley, M.R. et al., 2003, Mixing through oscillations and pulsations-A guide to achieving process enhancements in the chemical and process industries, Chem Eng Res Des, 81(A3): 373-383.

Oldshue, J.Y., 1983, Power correlations and effects of mixing environment, in Fluid Mixing Technology, 51-63 (Chemical Engineering McGraw-Hill Pub. Co., New York, USA).

Peng, C.Y., Lan, C.H. et al., 2006, Speciation and quantification of vapor phases in soy biodiesel and waste cooking oil biodiesel, Chemosphere, 65(11): 2054-2062.

Reis, N., Goncalves, C.N. et al., 2006, Application of a novel oscillatory flow micro-bioreactor to the production of gamma-decalactone in a two immiscible liquid phase medium, Biotechnology Letters, 28(7): 485-490.

Reis, N., Harvey, A.P. et al., 2005, Fluid mechanics and design aspects of a novel oscillatory flow screening mesoreactor, Chem Eng Res Des, 83(A4): 357-371.

Reis, N., Vincente, A.A. et al., 2004, Residence times and mixing of a novel continuous oscillatory flow screening reactor, Chem Eng Sci, 59(22-23): 4967-4974.

Schuchardt, U., Sercheli, R. et al., 1998, Transesterification of vegetable oils: a review, J Braz Chem Soc, 9(1): 199-210.

Schwab, A.W., Bagby, M.O. et al., 1987, preparation and properties of diesel fuels from vegetable-oils, Fuel, 66(10): 1372-1378.

Smith, K.B. and Mackley, M.R., 2006, An experimental investigation into the scale-up of oscillatory flow mixing in baffled tubes, Chem Eng Res Des, 84(A11): 1001-1011.

Stephens, G.G. and Mackley, M.R., 2002, Heat transfer performance for batch oscillatory flow mixing, Experimental Thermal and Fluid Science, 25(8): 583-594.

Stonestreet, P. and Van der Veeken, P.M.J., 1999, The effects of oscillatory flow and bulk flow components on residence time distribution in baffled tube reactors, Chem Eng Res Des, 77(A8): 671684.

Tan, R.R., Culaba, A.B. et al., 2004, Carbon balance implications of coconut biodiesel utilization in the Philippine automotive transport sector, Biomass \& Bioenergy, 26(6): 579-585.

Vicente, G., Martinez, M. et al., 2004, Integrated biodiesel production: a comparison of different homogeneous catalysts systems, Bioresource Technology, 92(3): 297-305.

\section{ACKNOWLEDGEMENTS}

The authors would like to thank to GlaxoSmithKline (GSK), Cambridge Overseas Trust, The Carbon Trust, and Prof. Brian Bellhouse for financially supporting different aspects of this project.

The manuscript was received 28 February 2007 and accepted for publication after revision 18 may 2007. 


\section{PSEP07030}

Queries

M. ZHENG, R. L. SKELTON and M. R. MACKLEY

Q1 Please list all authors.

Typesetter Queries 